\title{
Treatment of Multi-Drug Resistant Gram-Negative Bacterial Pathogenic Infections
}

\author{
Wardah Mohammad Akram (D), Godfred Antony Menezes* (D), Nida Abbas (D), \\ Wasim Ahmad (D) and Ahmed Mohamed Ahmed
}

RAK College of Medical Sciences (RAKCOMS), RAK Medical \& Health Sciences University (RAKMHSU), Ras Al Khaimah, UAE.

\begin{abstract}
The multidrug-resistant Gram-negative bacteria (MDR-GNB) infections in severely infected patients present numerous difficulties in terms of treatment failure where antibiotics cannot arrest such drug resistant bacteria. Based on the patient's medical history and updated microbiological epidemiology data, an effective empirical treatment remains critical for optimal results to safeguard human health. The aim of this manuscript is to review management of MDR-Gram negative pathogenic bacterial infections. Quick diagnosis and narrow antimicrobial spectrum require rapid and timely diagnosis and effective laboratories in accordance with antimicrobial stewardship (AS) principles. Worldwide, there is an increased emergence of Carbapenem-resistant Enterobacteriaceae (CRE), Pseudomonas aeruginosa, and Acinetobacter baumannii. Recently, novel therapeutic options, such as meropenem/vaborbactam, ceftazidime/avibactam, ceftolozane/tazobactam, eravacycline and plazomicin became accessible to effectively counteract severe infections. Optimally using these delays the emergence of resistance to novel therapeutic agents. Further study is required, however, due to uncertainties in pharmacokinetic/ pharmacodynamics optimization of dosages and therapeutic duration in severely ill patients. The novel agents should be verified for (i) action on carbapenem resistant Acinetobacter baumannii; (ii) action on CRE of $\beta$-lactam/ $\beta$-lactamase inhibitors dependence on type of carbapenemase; (iii) emergence of resistance to novel antibacterials and dismiss selective pressure promoting development of resistance. Alternative treatments should be approached alike phage therapy or antibacterial peptides. The choice of empirical therapy is complicated by antibiotic resistance and can be combated by accurate antibiotic and their combinations usage, which is critical to patient survival. Noteworthy are local epidemiology, effective teamwork and antibiotic stewardship to guarantee that medications are utilized properly to counter the resistance.
\end{abstract}

Keywords: Antimicrobial resistance, Enterobacteriaceae, Carbapenemase, Acinetobacter baumannii, bacteriophage

*Correspondence: godfred@rakmhsu.ac.ae; +971 543266244

(Received: March 03, 2020; accepted: August 28, 2020)

Citation: Akram WM, Menezes GA, Abbas N, Ahmad W, Ahmed AM. Treatment of Multi-Drug Resistant Gram-Negative Bacterial Pathogenic Infections. J Pure Appl Microbiol. 2020;14(3):1639-1647. doi: 10.22207/JPAM.14.3.02

(C) The Author(s) 2020. Open Access. This article is distributed under the terms of the Creative Commons Attribution 4.0 International License which permits unrestricted use, sharing, distribution, and reproduction in any medium, provided you give appropriate credit to the original author(s) and the source, provide a link to the Creative Commons license, and indicate if changes were made. 


\section{INTRODUCTION}

Gram-negative pathogens and antimicrobial resistance

Rising incidences of Gram negative bacteria (GNB) have become an immense problem worldwide as it may decrease the therapeutic choices considerably and renders anti-bacterial drugs ineffective. In Gram-negative pathogens, resistance has led into a principal cause of morbidity and mortality and a grave public health concern globally, specifically among Enterobacteriaceae family and non-fermenters ${ }^{1,2}$. The expansion of bacterial resistance has grown together with antimicrobial remedy from many years, but merely the GNB have recently begun to exhibit endurance to all regularly employed stages of antimicrobials. Clinicians have been enforced to contemplate different treatment modalities such as combinations of medications or even, to rediscover previous preparations, which had toxicity issues, in addition to suboptimal pharmacokinetics ${ }^{3}$.

The three most problematic $\mathrm{GNB}$; extended-spectrum $\beta$-lactamase (ESBL) producing Enterobacteriaceae, Acinetobacter baumannii, and Pseudomonas aeruginosa were identified by the 'antimicrobial availability task force' (these were a group of national experts that were responsible for development in concert with the upsurge in antibiotic resistance, as well as for reviewing trends in antibiotic research. Following this, they were asked to propose various resolutions to warrant the availability of efficient antibiotics in the near future $)^{4}$.

Various processes of antimicrobial opposition in GNB have been classified which include efflux pumps, target modification, hydrolyzing enzymes (e.g. $\beta$-lactamases). The widespread processes of resistance in GNB are firstly, the $\beta$-lactam and $\beta$-lactamase inhibitor arrangement such as monobactam (e.g. aztreonam), carbapenems and cephalosporins. Secondly, the $\beta$-lactam ring present in penicillin's gets hydrolyzed by $\beta$-lactamases ${ }^{5}$. Carbapenemresistance is utmost predominant in Acinetobacter spp., and in Pseudomonas spp. but in addition, it is constantly increasing in Enterobacteriaceae too, especially Klebsiella spp. and is an excellent marker for such situations ${ }^{1}$.
Acinetobacter baumannii is the usual carbapenem resistant pathogen linked to nosocomial infections worldwide and its infections are commonly seen to be occurring, especially in severely ill people with either major trauma, significant comorbidities, or immunosuppression. Carbapenem resistance among $A$. baumannii is conferred by multiple coexisting mechanisms, with production of $\beta$-lactamases being the predominant one ${ }^{6}$.

The recurrent resistance causes reduced therapeutic options, as well as higher mortality. $P$. aeruginosa is a problematic pathogen as it is hard to treat compared to other GNB. In P. aeruginosa, major role in carbapenem resistance is played by intrinsic/chromosomal-mediated resistance mechanisms. Typical resistance mechanisms in carbapenem resistant $P$. aeruginosa are variations or loss of the outer membrane proteins. These are required for the uptake of carbapenems, combined with overexpression of the chromosomal cephalosporinase AmpC $\beta$-lactamase, up regulation of efflux systems that can acquire resistance to unrelated antibiotic groups ${ }^{7}$.

The principal spp. among the cluster of carbapenem resistant Enterobacteriaceae (CRE) is Klebsiella pneumoniae ${ }^{8}$. The predominant cause of carbapenem resistance in $K$. pneumoniae is the generation of carbapenemases, which additionally affect other antibiotics such as, beta-lactam's. Noncarbapenemase-mediated process of resistance in carbapenem resistant $K$. pneumoniae is rare but, in general, vastly unknown ${ }^{1}$.

Intravenous treatment options for MDR GNB

Infections induced by multiple drug resistant (MDR) GNB microorganisms usually happen when there is hidden ailment, damage or hospitalization. MDR GNB might be obtained from different patients on broad-spectrum antibacterial agents for prolonged periods. Infections brought about due to MDR GNB are hard to treat thus may cause increasingly drawn out other effects, for example, pneumonia or septicemia. This can extend the duration of stay in the hospital, eventually leading to fatal outcomes. A few kinds of MDR GNB, for example, Acinetobacter spp. colonize in territories of the body with no apparent signs or signs and symptoms ${ }^{8,9}$. 
Groups of carbapenems like meropenem, imipenem and ertapenem have a variety of roles, one of them is the treatment of lethal infections with Extended Spectrum $\beta$-Lactamases (ESBL) and AmpC $\beta$-lactamase producing Enterobacteriaceae and they are used for empirical treatment of sepsis caused due to GNB. Imipenem has its effects during the emergence of resistance within $P$. aeruginosa but should be avoided in susceptible Pseudomonas spp. infections. If any resistance is found, exact levels of meropenem resistance should be immediately tested for and any sign of the accountable class of carbapenemase (e.g. $\mathrm{MBL} / \mathrm{KPC} / \mathrm{OXA} 48$-like) all imipenem or meropenem resistant isolates of Enterobacteriaceae should be detected. Preferably, meropenem and imipenem are chosen for empirical therapy of bacteremia (often developing within the urinary tract) because of their advantage to provide a broader spectrum range of treatment. Ertapenem is used in the advent of resistance via porin loss in ESBL- and AmpC $\beta$-lactamase producing Klebsiella spp. and Enterobacter spp. It is also used in a oncedaily dosing regimen for outpatient parenteral antimicrobial therapy of susceptible infections. In case the bacteria responsible for the infection eventually do not produce neither ESBLs nor AmpC $\beta$-lactamase, narrower-spectrum agents should be used instead of carbapenem $s^{8,9}$.

Ceftazidime is normally inadequate for treating infections with multi resistant Enterobacteriaceae, except certain OXA-48 carbapenemase producing bacterial strains. It remains helpful for infections caused by imipenem or quinolone susceptible $P$. aeruginosa strains. The utilization of ceftazidime in managing diseases caused due to ESBL-or AmpC $\beta$-lactamase producing Enterobacteriaceae or carbapenemaseproducing Enterobacteriaceae (CPE), besides for OXA-48 makers, regardless of whether in vitro tests recommendation is to be avoided ${ }^{10}$.

Ceftolozane/tazobactam is effective against various GNB, including Enterobacteriaceae and $P$. aeruginosa. These drugs have benefit in complicated cases of urinary tract infections (UTI's) and intra-abdominal infections. Ceftolozane is the most vigorous $\beta$-lactam against $P$. aeruginosa and it has potentially different uses. Concerning MIC, ceftolozane/tazobactam should be avoided in infections caused due to AmpC $\beta$-lactamase producing or KPC producing Enterobacteriaceae ${ }^{9}$. Co-amoxiclave is mixture of broad-spectrum antibiotic, amoxicillin and the $\beta$-lactamase inhibitor, clavulanic acid. It's efficacy is seen in lower tract UTIs triggered by ESBL-producing bacteria, particularly pathogens that lack coproduction of OXA-1 $\beta$-lactamase ${ }^{9}$.

Cefepime has a greater disappointment level in infections caused by ESBL-producing GNB than carbapenems, provided minimum inhibitory concentrations of (MICs) against cefepime are $\leq 1 \mathrm{mg} / \mathrm{L}$. The use cefepime for treatment of infection caused by ESBL or AmpC $\beta$-lactamase producing microorganism is not advised if inclined to the EUCAST breakpoint of MIC $\leq 1 \mathrm{mg} / \mathrm{L}$. The longer use of cefepime even at increased dose for isolates is not advised with (i) MIC of $2-8 \mathrm{mg} / \mathrm{L}$ (CLSI 'susceptible dose dependent') or (ii) MIC 2-4 mg/L (EUCAST intermediate) or (iii) strains producing both AmpC $\beta$-lactamase and ESBLs. Bacteremia caused due to $E$. coli strains barring ESBLs and with MIC $\geq 2 \mathrm{mg} / \mathrm{L}$ but $<8 \mathrm{mg} / \mathrm{L}$ can be correctly handled with cefepime but the use of cefepime is not advised to treat infections caused by $\mathrm{CPE}^{9}$.

Fluoroquinolones are advantageous in therapy of complicated UTI caused by Enterobacteriaceae residing in intestinal flora and additionally for quinolone-susceptible MDR GNB. Oral or intravenous (IV) fluoroquinolones can be used for the UTIs due to Enterobacteriaceae with ESBLs, if there is no conflict in vitro; then most ESBL producing strains are resistant to fluoroquinolones (comprising of levofloxacin and ciprofloxacin). Fluoroquinolones are used in combination with at least one of the other agents, trimethoprim/ sulfamethoxazole, ceftazidime or tigecycline to treat infections caused by Stenotrophomonas maltophilia, as resistance is usual. They can be used orally to handle UTI caused by susceptible MDR GNB ${ }^{10}$.

\section{Oral medications used for secondary or tertiary treatment including UTI}

The first agent that can be used orally is pivmecillinam, which is the oral formulation of mecillinam. They can be used uniquely to treat lower UTI that are caused by AmpC $\beta$-lactamase producing Enterobacteriaceae. This drug effectively combats ESBL-producing $E$. coli but not the carbapenemase producers. Individuals 
infected with these strains can be treated with carbapenems and then orally followed up on pivmecillinam alone for UTI due to mecillinam's apparent activity in vitro ${ }^{9}$.

The third generation cephalosporin known as cefixime is an oral formula, which, in contrast to pivmecillinam, is no longer effective by itself for ESBL-producing $E$. coli for the reason that there is resistance to more than one antibacterial agents, including quinolones. Cefixime and coamoxiclave combined can be used for treating ESBL producing Enterobacteriaceae, as supported by in-vitro data. These combinations must not be used against pathogens without investigations done to recognize AmpC $\beta$-lactamase and ESBL production. The transconjugant form of $E$. coli supports that cefixime in addition to clavulanate is convincing against strains producing CTX-M-15. Other cephalosporins such as cefdinir, ceftibuten and cefpodoxime, likewise indicated synergism with clavulanate, though sulbactam was less successful as a potentiator. Cefixime, not added or added to clavulanate, was not active against AmpC $\beta$-lactamase producing pathogens nor would it be relied upon to be active against Carbapenemaseproducing Enterobacteriaceae (CPE) ${ }^{9,10}$.

Nitrofurantoin is generally utilized in uncomplicated UTI. The resistance rate by $E$. coli is low, although new plasmid-intervened tools of defiance are now established. Low concentrations of the antibacterial agent reach the renal tissue and the circulation system but consequently, it is contraindicated if pyelonephritis (upper UTI) or bacteremia is suspected. Resistance of nitrofurantoin is intrinsic in Morganella morganii and Providencia spp. Furthermore, it is characteristic in Proteus spp. and Serratia spp. Moreover, antibacterial agent may not have an efficacy in the alkalinized urine that is due to urease-producing pathogens. For example, a lot of urease is produced by these and possibly Staphylococcus saprophyticus that is defenseless in vitro. Nitrofurantoin resistance is extremely common in $\mathrm{CPE}^{9}$.

Usually, there are no differences shown in fecal Enterobacteriaceae while consuming or following use of nitrofurantoin. Estimated GFR (eGFR) decreases with age and nitrofurantoin must no longer be utilized if it's $<45 \mathrm{~mL} / \mathrm{min}$. A short option of 3 to 7 days might be utilized in individuals with an eGFR of 30 to $44 \mathrm{~mL} / \mathrm{min} / 1.73 \mathrm{~m}^{2}$. Still, it is being used in lower UTIs with MDR organisms while the advantages of nitrofurantoin are expected to exceed the side effects. Repetitive or long-term plans of nitrofurantoin are related to serious pneumonic fibrosis ${ }^{9}$.

Fosfomycin is effective in the management of lower UTI caused by MDR Enterobacteriaceae. Fosfomycin in given orally either while fasting 2 or 3 hours prior to meals, as the absorption rate is minimized after the ingestion of food, which leads to drop in the concentrations in urine. Uncomplicated cystitis is the only indication for use of oral fosfomycin. The urinary concentration along with its constituents restricts $E$. coli multiplication for at least first 48 hours. Prophylactic regimens of pyelonephritis, in individuals with asymptomatic bacteriuria in pregnancy, and chronic prostatitis consist of oral fosfomycin trometamol. The long and repetitive use of fosfomycin has led to major complication such as plasmid as well as chromosomally mediated resistance in patients ${ }^{9}$.

The Next Invention of Agents and Adjuvants against GNB: Antibiotic Hybrids

The existence of the outer membrane makes Gram-negative microorganisms characteristically impervious to numerous antibacterial agents, particularly those with an increased molecular bulk and hydrophobicity. For example, lipopolysaccharide (LPS) structure makes the bacterial outer membrane extra prohibitive to hydrophobic antimicrobials, compared to the inner membrane. With this, we can confer that the hydrophilic carbohydrate part of LPS makes a hydration circle, which is impermeable to the track of hydrophobic molecules through the membrane. The glycopeptide antimicrobial, known as vancomycin, with a subatomic mass of $1449.3 \mathrm{~g} /$ mol, does not have any antibacterial action against most GNB. It prevents peptidoglycan synthesis by isolating the peptidoglycan precursors, so glycan cross-linking cannot be done. On the other hand, in gram-positive microbe's, vancomycin has no antibacterial restrictions, as its target is situated at the cell membrane. Nevertheless, it should cross the outer membrane and come to the periplasmic area to evoke its capacity in GNB. This is particularly difficult for vancomycin due 
to its defensive membrane barrier, hindering the membrane to be impermeable to all significant glycopeptide antimicrobials ${ }^{5}$.

It must be eminent that drug efflux overexpression may be problematic by causing intrinsic resistance to pathogens. It also affects the intracellular dosage of a therapeutic agent. Antibiotics from the oxazolidinone class, for example, linezolid, don't have potent action against most GNB, probably because of permeability obstructions over the outer membrane and/or efflux ${ }^{5,10}$

Pathogens are resistant to anti-microbial monotherapy because of their quick multiplying periods and high transmutation degrees. A few of them, for example, those from Mycobacterium spp. class show anti-microbial resilience because of their moderate growth. A mutation that presents general fitness under such antimicrobial stress is proliferated in enduring cells and along these lines offers the formation of a drug resistant approach. It is also evident that a few pathogens under antimicrobial monotherapy might prompt opposition systems that give cross-protection from other clinically irrelevant antibacterial classes $^{5}$. A hybrid antibiotic is defined as a synthetic, established agent capable of eliciting a desired antimicrobial effect and it is made up of two or more pharmacophores. This comprises of agents that are depicted as being either dualaction hybrids, antibiotic conjugates, chimeric, or multivalent/divalent ${ }^{5,11}$.

In the typical hybrid drug approach, a robust non-cleavable molecular linker capable of withstanding enzymatic and non-enzymatic attacks throughout in the body, covalently links the participating therapeutic agents. After arriving into the body, a hybrid drug is relied upon to provoke its antibacterial activity by using both of its pharmacophoric area at the same time. In any case, it ought to be noticed that the advancement of a hybrid medication that can concurrently restrain both targets by using just a particular molecular substance is a difficult task to achieve ${ }^{12}$.

The hybrid preparation approach is more prevalent than the prodrug approach because of scarce number of available bacteria specific cleavable linkers, in order to provide a prodrug delivery method. A hybrid agent is relied upon to stay a uni-molecular substance as it journeys to the site of infection and navigates through the bacterial membrane into the internal compartments such as periplasmic and cytosolic space. In any case, contrasts in such methodologies lie by the way they evoke their biotic function. A hybrid prodrug undergoes enzymatic cleavage, as it arrives in the bacterial cell, to yield two useful remedial elements, while a hybrid medication would stay a solitary substance all through its time course. In this way, hybrid medications might be beneficial as far as their drug metabolism and elimination is concerned $^{12}$.

Mostly, the antibiotic hydrids against GNB have a fluoroquinolone pharmacophore (for the most part ciprofloxacin). The decision of joining a fluoroquinolone as a principal medication might be credited to its strong substance properties that are constant even under numerous situations. Additionally, it might be simpler to attach fluoroquinolones to other helpful pharmacophore, for instance, $\beta$-lactams with slender areas of substance strength. In addition, the well-clarified structure-action association of fluoroquinolone antimicrobial and their expansive range of movement cause them to be an alluring class of anti-toxins $^{12}$.

The most encouraging passage is a combined naringenin/ciprofloxacin hybrid (known as hybrid 7) that has a strong antibiotic activity against Gram-positive microbes (MIC50 of $0.29 \mathrm{~g}$ / $\mathrm{ml}$ against Methicillin Resistant $S$. aureus [MRSA]), GNB (MIC50 of $0.71 \mathrm{~g} / \mathrm{ml}$ against MDR E. coli), and fungi (MIC50 of $0.14 \mathrm{~g} / \mathrm{ml}$ against amphotericin B-resistant Candida albicans) $)^{5,10,13}$.

Considering their apparent advantages, the idea of antibiotic hybrids is appealing but they are not without a problem. Stubborn chemical synthesis, molecular complexity and the effort required to institute the mode of action and advantage of hybrids over conventional medications make the approach of hybrids daunting ${ }^{10,13}$.

\section{Phage therapy/phage lytic proteins as} antimicrobial

Rise in multidrug resistant (MDR) clinical pathogens have led to an alarming drop in research and development of new antibiotics, and hence roles of reprogramming of antibiotics, finding alternatives and combinational therapies as future strategies in designing effective antibacterial 
agents are being exploited ${ }^{14-16}$. Alternatively, the use of bacteriophage therapy to treat bacterial infections is becoming increasingly popular in field of research. Phage technique, either as a substitute or as a supplement to antibiotic treatments, has proved to be extremely promising in two ways such as the identification of effective antibodies directed against pathogens, as well as for vaccine development. Recent clinical trials have shown great potential in conventional phage therapies that are based upon the mechanism to use naturally occurring phages, which pollute and lyse bacteria at their site of infection. Most importantly, their use has also been vastly studied for public health surveillance, as biosensor phages can be used to detect food and water contaminations, as well as prevent bacterial epidemics ${ }^{13}$.

How do bacteriophages perform their function?

Phages are naturally occurring, exceptionally varied, non-living biological beings containing DNA or RNA, surrounded inside a protein capsid. They are not able to reproduce independently and thus, are eventually dependent on bacterial host for survival. There are two steps as to how phages lyse the cell: first, the bacteriophage binds to a certain receptor on the bacterial cell surface. Then, they fuse this substance into the bacterial genome (known as temperate phages) either to duplicate vertically from mother to daughter cell or takeover the bacterial multiplication apparatus to produce the next phage descendants and lyse the cell (known as lytic phages). Immediately after the phage decedent's critical capacity has been reached, depending on the environmental aspects, the lytic proteins then become activated. They hydrolyze the peptidoglycan cell wall, thus releasing novel phage to restart the lytic cycle. ${ }^{12}$

During the lysis of the bacterial host there are two major protein classes engaged by the phage spp. The first is the transmembrane protein known as 'holin' and the second is a peptidoglycan cell wall hydrolase referred to as 'endolysin.' These work collectively in activating bacterial cell lysis. Advancements show that a newly noticed lysin, ABgp46, withholds the capability to lyse many gram-negative and MDR pathogens such as Salmonella Typhimurium, $A$. baumannii, $P$. aeruginosa, and Streptococcus pneumoniae, among others. This has led to the scientists discover that together, the phage lysins and antibiotics are more effective in abolishing infections in contrast to using antibiotics alone. This has further been proved by the use of in vitro and ex vivo phages in Clostridium difficile ${ }^{13}$.

Most of the time, phages are virulent only to those specific pathogens, which are carrying their complementary receptor, and this regulates lytic phage host range. The foremost usual lytic phages linked with pathogens infecting humans and the gut microbiotas are the Caudovirales (known as the tailed phages) and Microviridae. The tailed phages have double stranded DNA genomes whereas the Microviridae have single-stranded viruses $^{13}$.

Contrastingly, lysogenic phages can also be of importance to the bacterial host as the phages incorporate their genetic composition into the bacterial chromosome. This results in encoding for virulence factors (e.g., botulinum toxin, diphtheria toxin and shiga toxin), and antibiotic resistance genes (e.g., $\beta$-lactamases) ${ }^{13}$.

There is another therapy known as 'conventional phage therapy.' This solemnly focuses on strictly lytic phages that only kill the bacterial host. To treat animals, lytic phages are collected into "phage cocktails" which contain numerous phages that have in vitro effectiveness against the bacterial pathogen ${ }^{13}$.

\section{Phage lytic proteins as antimicrobials}

Current research has used bacteriophages on animal prototypes to examine a range of clinically significant pathogens. The following pathogens have been examined:

- A single dose of phage cocktail was sufficiently used as prophylaxis in $C$. difficile induced ileocecitis, while the control animals obtaining clindamycin passed away within 96 hours. Phage cocktails also drastically compacted C. difficile growth in vitro and restricted multiplication in vivo.

- On oral administration of phage cocktail for patients suffering from gut-derived sepsis, the mortality rate reduced to $66.7 \%$.

- A single strain of phage administered intraperitoneally was adequate to protect vancomycin-resistant E. faecium, ESBL producing $E$. coli, as well as imipenemresistant $P$. aeruginosa.

- Phage cocktails are used to treat antibiotic- 


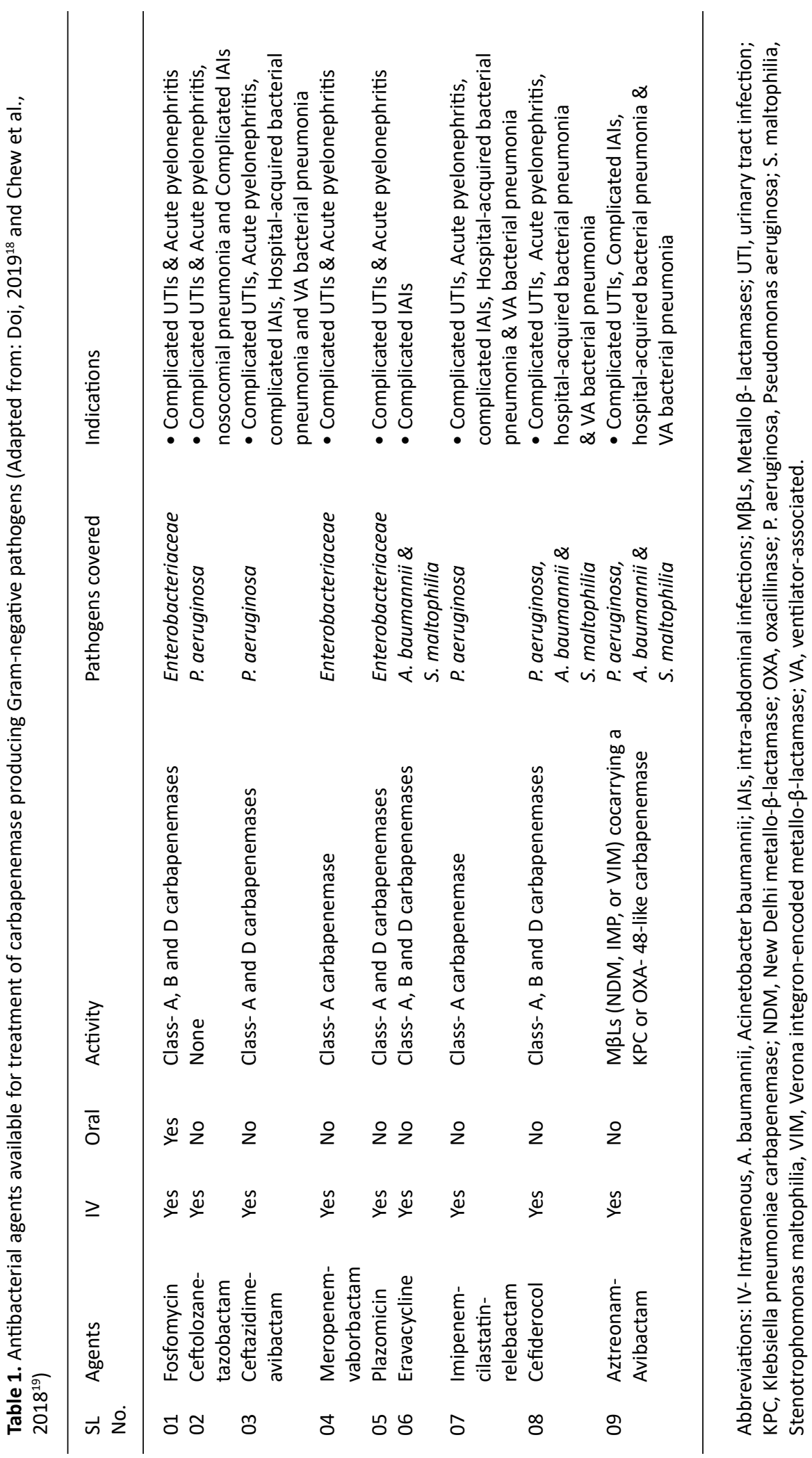


resistant $P$. aeruginosa contaminations of the lungs, gastrointestinal tract and skin.

Further studies show likewise favorable outcomes for multidrug-resistant $E$. coli, Vibrio parahaemolyticus, S. aureus, and A. baumannii. These researches have shown that there is a sign that bacteriophages will be able to restore antibiotic sensitivity in antibiotic resistant bacteria, such as multidrug-resistant $P$. aeruginos $a^{12,13}$.

Trials on humans have also been conducted using bacteriophages. They have vastly been used in clinical treatment of everyday bacterial pathogens such as E. coli, S. aureus, Streptococcus spp., P. aeruginosa, Proteus spp. Both, therapeutic and prophylactic effectiveness has been achieved in cases of surgery and gastroenterology. Recently, a study was conducted where six patients with diabetic foot ulcers unresponsive to antibiotics, received topical application of $S$. aureus - specific phage. The results showed that this was sufficient for recovery in all patients ${ }^{13}$.

\section{Biosensor phages}

Currently, none of the phage therapy products have been permitted for use to humans in the European Union or The States. Nevertheless, the case is different in food industry. In this, numerous commercial phage formulations are used for biocontrol of bacteria and the FDA approves them by being listed under the categorization called "generally considered as safe." The formulations are under use against MRSA, Salmonella spp., E. coli, Listeria monocytogenes, Mycobacterium tuberculosis, Pseudomonas syringae and Campylobacter spp. These recent studies have suggested that bacteriophage play a strategic role in successfully refining food safety at numerous stages such as in food production and processing, while also reducing the bacterial contamination in vegetables, fruits and dairy products. However, additional study is still required in this aspect ${ }^{13}$.

Bioengineered chimeras of phage-derived lytic proteins may be the new era of antibacterials

Chimeric lysine forms by linking the active site of a lysin with a cell wall binding domain. Chimeric lysines are highly skilled of saving animals infected with MRSA bacteremia. They have efficiently been able to avert fatality from $S$. pneumoniae and stop growth of methicillin-sensitive Staphylococcus aureus
(MSSA) endophthalmitis. However, researches on such bioengineered proteins are still in the early stages $^{13}$.

GNB have an impermeable LPS outer membrane; whereas lysins perform through enzymes. They cleave the bacterial cell wall thus, becoming less successful against such microorganisms. As an effort, to overcome its target, bioengineered artificial lysin molecules, termed artilysins, have been manufactured which are able to penetrate the outer membrane. Until now in a nematode gut model, artilysins have successfully decolonized $P$. aeruginos $a$ as well as protected the human keratinocytes when tested with $A$. baumannii ${ }^{13}$.

Antibacterial agents available for treatment of multidrug resistant (MDR) Gram-negative pathogens

To summarize the antibacterial agents, available for treatment of multidrug resistant (MDR) Gram-negative pathogens include, amikacin; amoxicillin/clavulanate; ampicillin/sulbactam; aztreonam; cefepime; cefixime and other oral cephalosporins; ceftazidime; ceftazidime/ avibactam; ertapenem; fluoroquinolones; fosfomycin; gentamicin; imipenem and meropenem; nitrofurantoin; piperacillin/ tazobactam; pivmecillinam; polymyxins (including colistin); temocillin; tigecycline; tobramycin \& trimethoprim/sulfamethoxazole. The antibacterial agent used depends on antibiotic policies, empirical use, toxicity and mechanisms of antimicrobial resistance ${ }^{17-19}$ (Table 1).

\section{CONCLUSION}

The choice of empirical therapy is complicated by antibiotic resistance and can be combated by accurate antibiotic and their combinations usage, which is critical to patient survival. Noteworthy are local epidemiology, effective teamwork and antibiotic stewardship to guarantee that medications are utilized properly to counter the resistance.

\section{ACKNOWLEDGMENTS}

Not applicable.

\section{CONFLICT OF INTEREST}

The authors declare that there is no conflict of interest. 


\section{AUTHORS' CONTRIBUTION}

All authors listed have made a substantial, direct and intellectual contribution to the work, and approved it for publication.

\section{FUNDING}

None.

\section{ETHICS STATEMENT}

This study was approved by RAKMHSU-

REC.

\section{DATA AVAILABILITY}

All datasets generated or analyzed during this study are included in the manuscript.

\section{REFERENCES}

1. El-Kholy AA, Girgis SA, Shetta MAF, Abdel-Hamid $\mathrm{DH}$, Elmanakhly AR. Molecular characterization of multidrug-resistant Gram-negative pathogens in three tertiary hospitals in Cairo, Egypt. Eur J Clin Microbiol Infect Dis. 2020;17:987-992. doi: 10.1007/s10096-02003812-z

2. Theuretzbacher U. Global antimicrobial resistance in Gram-negative pathogens and clinical need. Curr Opin Microbiol, 2017;39:106-112. doi: 10.1016/j. mib.2017.10.028

3. Bassetti M, Peghin M, Vena A, Giacobbe DR. Treatment of Infections Due to MDR Gram-Negative Bacteria. Frontiers in Medicine. 2016;6:74. doi: 10.3389/ fmed.2019.00074

4. Talbot GH, Bradley J, Edwards JE, Gilbert D, Scheld M, Bartlett JG. Bad Bugs Need Drugs: An Update on the Development Pipeline from the Antimicrobial Availability Task Force of the Infectious Diseases Society of America. Clin Infect Dis. 2006;42(5):657-668. doi: $10.1086 / 499819$

5. Antimicrobial Resistance in Gram-negative Bacteria: a Continuous challenge. https://www.medlabme. com/magazine/en/diagnostic-articles/antimicrobialresistance-in-gram-negative-bacteria-a-continuouschallenge.html, accessed 19 April,2019.

6. Dahdouh E, Gomez-Gil R, Pacho S, Mingorance J, Daoud Z, Suarez M. Clonality, virulence determinants, and profiles of resistance of clinical Acinetobacter baumannii isolates obtained from a Spanish hospital. PLOS ONE. 2017;12(4):e0176824. doi: 10.1371/ journal.pone.0176824

7. Thaden JT, Park LP, Maskarinec SA, Ruffin F, Fowler VG, Duin DV. Results from a 13-Year Prospective Cohort Study Show Increased Mortality Associated with Bloodstream Infections Caused by Pseudomonas aeruginosa Compared to Other Bacteria. Antimicrob Agents Chemother. 2017;61(6):e02671-16. doi: 10.1128/AAC.02671-16
8. Satlin MJ, Chen L, Patel G, et al. Multicenter Clinical and Molecular Epidemiological Analysis of Bacteremia Due to Carbapenem-Resistant Enterobacteriaceae (CRE) in the CRE Epicenter of the United States [Internet]. Antimicrob Agents Chemother. American Society for Microbiology Journals. 2017. https://aac.asm.org/content/61/4/e02349-16. doi: 10.1128/AAC.02349-16

9. Hawkey P, Warren RE, Livermore DM, et al. Treatment of infections caused by multidrug-resistant Gramnegative bacteria: report of the British Society for Antimicrobial Chemotherapy/Healthcare Infection Society/British Infection Association Joint Working Party. J Antimicrob Chemother. 2018;73(suppl3). doi: $10.1093 / \mathrm{jac} / \mathrm{dky027}$

10. Levison ME, Kaye D. The ABCs of antimicrobial drug resistance in gram-negative bacilli Page 1 [Internet]. Healio.com. 2016. https://www.healio. com/infectious-disease/antimicrobials/news/print/ infectious-disease-news/\%7Bfc3bd216-98b1-411e8ea3-7757dcd1d89b\%7D/the-abcs-of-antimicrobialdrug-resistance-in-gram-negative-bacilli?page=1.

11. Lin D, Koskella B, Lin H. Phage therapy: An alternative to antibiotics in the age of multi-drug resistance. World Journal of Gastrointestinal Pharmacology and Therapeutics. 2017;8(3):162-173. doi: 10.4292/wjgpt. v8.i3.162

12. Criscuolo E, Spadini S, Lamanna J, Ferro M, Burioni R. Bacteriophages and Their Immunological Applications against Infectious Threats. Journal of Immunology Research. 2017;2017:1-13. doi: 10.1155/2017/3780697

13. Domalaon R, Idowu T, Zhanel GG, Schweizer F. Antibiotic Hybrids: the Next Generation of Agents and Adjuvants against Gram-Negative Pathogens? Clin Microbiol Rev. 2018;31(2). doi: 10.1128/cmr.00077-17.

14. Tiwari R, Dhama K, Chakraborty S, Kumar A, Rahal A, Kapoor S. Bacteriophage therapy for safeguarding animal and human health: A review. Pak J Biol Sci. 2014;17(3):301-315. doi: 10.3923/pjbs.2014.301.315

15. Leon-Buitimea A, Garza-Cardenas CR, Garza-Cervantes JA, Lerma-Escalera JA, Morones-Ramirez JR. The Demand for New Antibiotics: Antimicrobial Peptides, Nanoparticles, and Combinatorial Therapies as Future Strategies in Antibacterial Agent Design. Front Microbiol. 2020;11:1669. doi: 10.3389/ fmicb.2020.01669

16. Dubey KK, Indu, Sharma M. Reprogramming of antibiotics to combat antimicrobial resistance [published online ahead of print, 2020 Aug 10]. Arch Pharm (Weinheim). 2020;e2000168. doi: 10.1002/ ardp.202000168

17. Doi Y. Treatment Options for Carbapenem-resistant Gram-negative Bacterial Infections.Clin Infect Dis. 2019;69(Suppl.7):S565-S575. doi: 10.1093/cid/ciz830

18. Chew KL, Tay MKL, Cheng B, Lin RTP, Octavia S, Teo JWP. Aztreonam-Avibactam Combination Restores Susceptibility of Aztreonam in Dual-CarbapenemaseProducing Enterobacteriaceae. Antimicrob Agents Chemother. 2018;62(8):e00414-18. doi: 10.1128/ AAC.00414-18 